\title{
EL DESARROLLO DE COMPETENCIAS DOCENTES PARA EL FORTALECIMIENTO DE LA CALIDAD EDUCATIVA EN LA ENSEÑANZA SUPERIOR.
}

\section{THE DEVELOPMENT OF TEACHING COMPETENCES FOR THE STRENGTHENING OF EDUCATIONAL QUALITY IN HIGHER EDUCATION.}

\author{
Nadia Aurora González Rodríguez, MsC. \\ Máster en Ciencias de la Educación (Cuba). \\ Docente Titular de la Facultad de Marketing y Comunicación, \\ Coordinadora de Publicaciones de la Universidad Tecnológica ECOTEC, Ecuador. \\ ngonzalez@ecotec.edu.ec
}

\section{ARTÍCULO DE REFLEXIÓN}

Recibido: 9 de septiembre de 2019.

Aceptado: 25 de octubre de 2019.

\section{RESUMEN}

La calidad en la educación es fundamental para direccionar los procesos sustantivos de este nivel de enseñanza de gran importancia para la evolución de un país, ya que forma a los futuros profesionales de una nación. Una de las asociaciones que se realizan en cuanto a este término es con la excelencia, lo cual es difícil de lograr, pero no imposible. Por esta razón, la presente investigación tiene como objetivo fundamental proponer estrategias metodológicas para el desarrollo de competencias docentes como forma de contribución a la mejora de la calidad educativa en el nivel universitario. Esta investigación es de tipo descriptiva con un enfoque cualitativo ya que se utilizaron métodos teóricos como el análisis-síntesis e inducción-deducción, como forma de procesar la información pertinente en las fuentes de información sobre el problema en relación con la alternativa de solución para la puesta en práctica en Instituciones de Educación Superior. La propuesta presentada asume la experiencia didáctica de la investigadora debido a los favorables resultados prácticos de su aplicación durante el proceso docente, el cual constituye uno de los ejes estratégicos más importantes, en el marco de esta enseñanza. 
Palabras clave: educación superior, calidad educativa, competencias docentes, estrategia metodológica.

\section{ABSTRACT}

Quality in education is essential to address the substantive processes of this level of education of great importance for the evolution of a country, since it trains the future professionals of a nation. One of the associations that are made regarding this term is with excellence, which is difficult to achieve, but not impossible. For this reason, this research has as a fundamental objective to propose methodological strategies for the development of methodological teaching competencies as a way of contributing to the improvement of educational quality at the university level. This research is descriptive with a qualitative approach since theoretical methods such as analysissynthesis and induction-deduction were used, as a way of processing the relevant information in the sources of information about the problem as an alternative application in Higher Education Institutions. The proposal presented assumes the didactic experience of the researcher due to the favorable practical results of its application during the teaching process as one of the most important strategic axes in the framework of this teaching.

Keywords: higher education, educational quality, teaching skills, methodological strategy.

\section{INTRODUCCIÓN}

En el contexto de la educación superior actual existe la necesidad de que los profesores fortalezcan sus competencias docentes, en función del incremento de la calidad académica. Muchos son los motivos que sustenta esta necesidad; pero uno de los desafíos lo constituye la formación inicial de estos profesionales, quienes en su mayoría no recibieron una formación pedagógica; sino que su preparación estuvo centrada en la ciencia. En tal sentido, constituye un eslabón primordial para su presente accionar profesional, desarrollar competencias docentes en el profesor universitario que facilite la transmisión de estos conocimientos mediante capacitaciones donde se articule la experticia profesional con en el complejo accionar de la práctica pedagógica. 
Este interés parte de la observación a docentes de nuevo ingreso, cuyas evaluaciones evidencian una clara necesidad de brindar la debida atención a esta dificultad; con el fin de evitar el fracaso estudiantil y elevar los niveles de retención en este nivel. Estos problemas son el resultado de estos profesores no cuentan con las habilidades básicas pedagógicas para impartir los conocimientos sobre su ciencia. Si bien es cierto que, a inicios de la práctica académica, siempre es complicado la ejecución del proceso de enseñanza-aprendizaje, cuando existe una previa formación pedagógica el camino se hace más certero.

Muchos docentes universitarios comienzan su desempeño poniendo en práctica su experiencia como estudiantes y van adquiriendo con la práctica, las competencias docentes necesarias mediante la sistematización y mejora progresiva de esta actividad. En cambio, esta forma de proceder no es la manera más adecuada, ya que por el camino existen tropiezos que podrían ser evitados si contaran con las competencias docentes necesarias para que su experiencia no fuera desafortunada.

En la actual sociedad del conocimiento, para que los futuros profesionales sean más capaces de enfrentar con proactividad los retos del mundo laboral, necesitan de una orientación eficaz de sus principales guías: los docentes universitarios. En cambio, en ocasiones esta práctica se realiza de manera empírica basada en su experiencia como estudiante y se convierte en una ejecución parcelada, algunas veces desarticulada, descontextualizada o con pretendidas habilidades; ya que su formación no ha sido propiamente pedagógica, lo cual repercute en la correcta formación del universitario.

Esta investigación debe hacer referencia a los antecedentes sobre el interés que las políticas educativas y la gestión en la educación superior, como forma de propiciar el crecimiento de las Universidades a nivel Internacional. Por un lado, la "Conferencia Mundial sobre la Educación Superior para el Siglo XXI" (París, noviembre de 1998), se realizó en un momento propicio debido a la influencia de las política de los estados y los organismos multilaterales de financiamiento, como el Banco Mundial (Tünnermann, 2010). Por otro lado, tal como se evidencia en el contexto europeo, los cambios que promueve el proceso de Convergencia desde la Declaración de Bolonia en 1999, que más directamente afecta al estudiante universitario europeo, es la transformación del binomio aprendizaje-enseñanza; pero que también tuvieron importantes efectos en América Latina y el Caribe como un tratado que revolucionó este nivel de enseñanza (Pozo, Bretones, Martos, y Alonso, 2011; Lago, Gamoba, y Montes, 2014). 
En el contexto de la Educación Superior en Ecuador, para asegurar la calidad, las políticas educativas pasaron por transformaciones importantes. A inicios del año 1998 se renueva la Constitución Política del Ecuador para atender la problemática universitaria vigente; así como la Ley Orgánica de Educación Superior, LOES (2000). (Sánchez, Chávez, y Mendoza, 2018). Asimismo, esta Ley señala un aspecto importante en cuanto a formación académica:

Art. 4.- Derecho de la Educación Superior. - El Derecho a la educación superior consiste en el ejercicio efectivo de la igualdad de oportunidades en función de los méritos respectivos, a fin de acceder a una formación académica y profesional con producción de conocimiento, pertinente y de excelencia (LOES, 2010).

De esta manera se puede apreciar el interés que se le ha brindado durante décadas a la Educación Superior, puesto que no por ser parte de la culminación de etapa estudiantil, debe ser concebida como un nivel de menor importancia. Si se parte de la premisa de que el ser humano desde que nace hasta que muere está en constante aprendizaje, entonces se podría afirmar que la Universidad es un eslabón fundamental en el proceso de formación del ser humano.

Por esta razón se plantea la siguiente interrogante científica: ¿Cómo contribuir al fortalecimiento de la escritura académica en los estudiantes de la educación superior? Para dar respuesta a esta pregunta se presenta el siguiente objetivo general: proponer estrategias metodológicas mediante capacitaciones que fortalezcan el desarrollo de competencias docentes de los académicos universitarios para la mejora de la calidad educativa en este nivel de enseñanza.

Esta investigación se ha desarrollado con un enfoque cualitativo de tipo descriptiva, para su desarrollo de utilizaron métodos teóricos, tanto el análisis-síntesis como la inducción-deducción, en función de revisar los diferentes puntos de vista más pertinentes según expertos, que propicien el desarrollo de competencias docentes como vía para elevar la calidad educativa en las Universidades. Este procedimiento constituye una forma útil de constatar la diversidad de concepciones sobre esta preocupación, que constituye uno de los eslabones fundamentales para el eje sustantivo de las Instituciones de Educación Superior de cualquier país a nivel nacional e internacional; lo cual tributa en la formación pertinente de los futuros profesionales de cualquier nación. 


\section{REVISIÓN TEÓRICA}

\section{La educación superior, su misión y visión institucional.}

La educación superior cumple con una misión social de formar individuos integrales que sean proactivos y responsables de su contexto social; con una misión y visión comprometida con la conservación de su realidad, preocupación por su aprendizaje autónomo para dirigir su formación hacia la pertinencia de las demandas actuales de empleo (Lago, Gamoba, y Montes, 2014). Por lo antes expuesto, los docentes tienen nuevos desafíos que afrontar en su desempeño docente, para que este tribute a la eficacia profesional de sus estudiantes, futuros profesionales del mañana. Asimismo, es de vital importancia destacar el papel transcendental de la gestión académica en los procesos de evaluación de sus docentes, puesto que los resultados son un marcador importante para proyectarse hacia la mejora de las competencias docentes.

Por otro lado, aspectos como la investigación, movilidad docente y estudiantil, tecnología e innovación educativa son importantes para que las actuales Instituciones de Educación Superior (IES) enfoques sus modelos pedagógicos y propicien el desarrollo de competencias docentes en sus académicos. De igual manera, es imprescindible concebir un modelo de profesor que propicie el respeto, la comunicación empática y asertiva hacia sus estudiantes; con el fin de promover un aprendizaje significativo basado en la adecuada relación interpersonal. Leiva (2011) citado en Lago, Gamoba, y Montes (2014) señala: "las estrategias metodológicas y evaluativas, sobre todo con los principios, valores, convicciones y traducción práctica de los mismos, desde la perspectiva de la coherencia entre la teoría y práctica del docente y entre lo que dice y hace" (p. 175).

Luego de los antes mencionado, se debe pensar en la calidad de la educación asociado con la eficiencia del rendimiento hacia la eficiencia social, pero que debe tener en consideración los sistemas actuales de baja calidad y el contexto de la educación (Martin, 2018). La calidad está relacionada con la repercusión internacional en cuanto al intercambio de conocimiento (UNESCO, 1998). Para ello "las nuevas tecnologías de la información tienen un gran impacto en la adquisición de conocimientos teóricos y prácticos" (Ortega y González, 2017, p. 13); lo cual se relaciona con el proceso de investigación de los docentes para su preparación como profesionales de su ciencia y de los adelantos didácticos y metodológicos en función de una mejor práctica docentes. 
En el contexto universitario en la región de América Latina y el Caribe se ha evidenciado un incremento sustancial de la matrícula, del $21 \%$ a inicios de 2000 al $43 \%$ a finales de 2013 , lo que representa más de 20 millones de estudiantes que pertenecen a 10.000 instituciones de educación superior de toda la región (Sandoval, 2017). Por otro lado, es importante considerar que las IES deben estar respaldadas por las políticas educativas de sus países. A decir de los siguientes expertos como (Ferreyra, Avitabile, Botero, Hamovich, y Urzúa, 2017):

(...) los hacedores de política deben ser conscientes de los retos y limitaciones de las políticas de educación superior, y de las disyuntivas existentes entre el acceso a la educación superior y la graduación, ya que el primero tiene el potencial de perjudicar a la segunda (p.34).

También, a decir de (Enríquez, 2006): "La universidad debe enseñar a pensar, ejercitar el sentido común y dar rienda suelta a la imaginación creadora" (p.2). El reto se encuentra en propiciar la relación entre las necesidades sociales y los intereses políticos del contexto. A decir de (Orozco, 2010) existen actualmente diversas preocupaciones sobre todo por parte de los gobiernos en relación a la educación superior:

a) la politización de los consejos o comisiones nacionales encargadas de adelantar los procesos de acreditación; b) que la lucha por la calidad se vuelva contra las personas de menores ingresos por no poder acceder a centros de excelencia, los cuales, para mantener sus niveles de exigencia, se inclinan a trabajar sólo con estratos de ingresos altos; c) los costos crecientes del funcionamiento de los sistemas en cada país, y d) la carencia de sistemas de información que garanticen que ésta llegue a los usuarios finales de los servicios educativos (p.31).

De esta manera se puede deducir que todavía el acceso a la universidad para todos es un factor que aún preocupa a las naciones, puesto que de ello depende el progreso de los países, sobre todo para aquellos en vía de desarrollo. Está en las manos de las presentes generaciones establecer estrategias para el cambio oportuno de esta situación para contribuir a la extensión universitaria de los que son mayoría.

\section{La calidad educativa en el contexto de la Educación Superior.}

La calidad de la enseñanza universitaria es fundamental para ejercer transformaciones directas como resultado de evaluaciones internas que propicien la mejora de los procesos formativos en 
este nivel educativo, no menos importante para un país y su sociedad. De igual manera, en el contexto tanto mundial como Latinoamericano, se ha evidenciado la aplicación de este concepto de diferentes maneras por parte de las instituciones y el sistema educativo. No obstante, esta se entenderá como la aplicación de requisitos y reglamentos, como puede ser en "términos de costobeneficio, infraestructura avanzada y optimización de recursos, eficiencia terminal y calificación del personal académico" (González y Santamaría, 2013, p. 133).

Por otro lado, las universidades de América Latina y el Caribe, no han estado exentas de esta preocupación sobre este binomio y una de las formas de control de este proceso como tendencia mundial ha sido la acreditación de la educación superior, que comenzó por la década de los 90 como forma de rendir cuenta sobre el gasto público en relación con el desempeño académico de sus Instituciones; aunque más tarde se extendió al resto de las universidades, como un proceso voluntario (Martínez, Tobón, y Romero, 2017). De igual forma, estos procesos de mejoras necesitan de retroalimentación para centrar su atención más en los perfeccionamientos de las prácticas educativas y no tanto en los procesos administrativos que no son el eslabón fundamental de la evolución de la educación en función del desarrollo social de un país.

Al respecto, existen diversos criterios sobre la experticia de un docente universitario, en su contribución a la calidad en la educación superior, según Guzmán (2011) quien se basa en diferentes fuentes resumidas a continuación: a) Dominio amplio y especializado de la disciplina que enseña; b) Dominio pedagógico general; c) Dominio pedagógico específico del contenido; d) Dominio curricular; e) Claridad acerca de las finalidades educativas; f) Ubicarse en el contexto o situación donde enseña; g) Conocimientos de los alumnos y de los procesos de aprendizaje; $h$ ) Conocimiento de sí mismo; i) Sentimiento de auto-eficacia; j) Método experencial. En este sentido, es bastante abarcador concentrar en una sola persona todas estas habilidades, pero de ello depende una mejor proyección hacia la excelencia educativa y la satisfacción de los estudiantes.

La calidad educativa no es nada sin la evaluación de los procesos, por ellos cuando se produce la verificación de los resultados, es imprescindible planificar las maneras apropiadas para su ejecución. En este sentido, se debe tomar en consideración tres aspectos importantes: la superestructura, la estructura y la infraestructura; la primera entendiéndose por la misión, visión, objetivos, finalidades, perfil del egresado y modelo educativo; en cambio, la segunda es la organización académica de la institución con sus actores en relación con los planes de estudio, las líneas de los proyectos de investigación, las acciones de extensión y transferencia-difusión 
de conocimiento; y a última referida a los recursos materiales, financieros y servicios (Álvarez, Chaparro, y Reyes, 2015).

En cuanto a la enseñanza-aprendizaje, se pueden establecer diferentes criterios asociados con el desarrollo profesional: formación e instrucción, según la concepción del Modelo de Competencias Profesionales (MCP) se pueden identificar las siguientes:

Cuadro 1. Modelo de Competencias Profesionales (MCP).

\begin{tabular}{|c|c|}
\hline ELEMENTOS & DESCRIPCIÓN \\
\hline Aprendizaje & $\begin{array}{l}\text { Significativo para la solución de problemas de manera creativa de } \\
\text { acuerdo a contextos diferentes. }\end{array}$ \\
\hline Experiencia práctica & $\begin{array}{l}\text { Escenarios reales para la aplicación de la teoría demostrando } \\
\text { competencias en el desempeño de la profesión. }\end{array}$ \\
\hline Papel activo del docente & $\begin{array}{l}\text { Sistemática actualización de los estilos de aprendizaje de sus } \\
\text { estudiantes, para concebir ambientes de aprendizajes, guiar, } \\
\text { supervisar y evaluar el programa académico y la práctica de los } \\
\text { alumnos. }\end{array}$ \\
\hline Papel activo del estudiante & $\begin{array}{l}\text { Participante dinámico con altas expectativas y con una visión de } \\
\text { aprendizaje para toda la vida. }\end{array}$ \\
\hline Infraestructura estimulante & $\begin{array}{l}\text { Espacios científicos, tecnológicos, culturales y deportivos } \\
\text { preparados y suficientes. }\end{array}$ \\
\hline $\begin{array}{l}\text { Diversidad de estrategias de } \\
\text { enseñanza }\end{array}$ & $\begin{array}{l}\text { Talleres, seminarios, casos de estudio, proyectos, ensayos, } \\
\text { equipos multidisciplinarios, planteamientos de problemas, clases } \\
\text { en idiomas extranjeros combinando lo cognitivo, afectivo y } \\
\text { conductual. }\end{array}$ \\
\hline Evaluación & $\begin{array}{l}\text { Desempeño individual, grupal o en equipo para una visión } \\
\text { holística manteniendo sus etapas de diagnóstico, formativa y } \\
\text { sumativa; con fuentes de evidencia y criterios de desempeño } \\
\text { indicados al comienzo de los cursos, en mutuo acuerdo entre los } \\
\text { implicados en el proceso. }\end{array}$ \\
\hline
\end{tabular}

Fuente: Elaboración propia a partir de Quiroz (2007). 
Lo antes expresado debe incluirse en el proceso de gestión de la calidad educativa, en función de propiciar la mejora del desempeño, tanto de estudiantes como docentes. En tal sentido, el currículo flexible debe ser un eslabón fundamental de la aplicación de estos elementos indispensables a tomar en consideración por la IES, teniendo en cuenta el contexto del país. Como afirma (Murillo, 2011) a nivel del centro educativo "los conceptos de «cultura de mejora», «procesos de mejora» y «resultados de mejora» son esenciales" para la proyección acertada para propiciar la calidad educativa y si bien es cierto, estos conceptos se pueden aplicar en cualquier nivel educativo; en cambio, es necesario contar con docentes capacitados para desempeñar este proceso y una de las formas para lograrlo es fortaleciendo sus competencias docentes.

\section{Las competencias docentes en la actualidad universitaria.}

La educación superior en la región de América Latina y el Caribe tiene el gran reto de continuar con su universalización mediante los recursos ilimitados propiciados por la tecnología. Los educadores de este nivel de enseñanza, deben brindarle la importancia que requiere al desarrollo de sus competencias docentes, con el mismo interés de los niveles anteriores. "Este paradigma impone condiciones de exigencias respecto a la competitividad y vigencia de los conocimientos de los profesionales graduados" (Fernandez, Sotolongo, y Martínez, 2016, p. 9).

En ocasiones, algunos docentes suelen concentrar su interés profesional en el conocimiento de la ciencia de su formación, lo cual es fundamental para una eficiente práctica académica; sin embargo, también es oportuno resaltar la importancia de contribuir a la formación de un académico capacitado con destrezas imprescindibles para viabilizar de la mejor manera los conocimientos a sus discentes. Por cuanto, para el desarrollo de un docente universitario, es fundamental relacionar dos tipos de conocimiento, uno vinculado con la disciplina y el otro con el pedagógico (Nemiña, García, y Montero, 2009).

Asimismo, es de vital importancia la motivación y vocación que se siente ante la práctica docente, el sentido de pertenencia y la responsabilidad social de este desempeño. Además, la adquisición de competencias está relacionada con las cualidades de la persona, tales como, conocimientos, habilidades, actitudes y valores prestos a la resolución de problemas en tiempo real (Palominos, Mendez, y Barrera, 2014). Con motivo de lo antes expuesto, (Silander y Stigmar, 2018), afirma que una manera de afrontar las carencias de experiencias pedagógicas es mediante la planificación de capacitaciones asociadas con la formación docente. Sin embargo, es interesante 
indicar la necesidad de articulación entre estos cursos con su aplicación en la práctica docente y los controles de mejora del rendimiento académico de los estudiantes.

Por tanto, se hace más complejo los procesos de evaluación de los sistemas de Calidad Educativa Universitaria y una de las formas más apropiadas es comprobando los modelos teóricos, de acción docente mediante la observación de los resultados, no solo individuales y a corto plazo, sino como resultados de aprendizajes apreciables para la sociedad y de forma inmediata para su aplicación en la realidad socio-profesional-laboral, con el fin de mejorar la calidad de vida de su contexto social asociado con la internalización, el desarrollo regional, tecnológico y reformas educativas; como variable determinante para la política laboral y problemática empresarial (García, 1999; Jiménez, Palmero, y Jiménez, 2012; Tejada, 2013).

En el contexto de la educación superior de los países desarrollados, la dimensión del desarrollo profesional docente, cobra un valor de excelencia; pero son conscientes de la relevancia que este aspecto tiene para la calidad de la educación. Según la Comisión Europea, 2008 citado en Tejada (2008, p. 176) existen tres niveles de competencias relevantes para los docentes:

- Competencias profesionales: relacionadas con la experiencia laboral y las destrezas técnicas profesionales; tienen que ver con las competencias específicas de su especialidad.

- Competencias pedagógicas y sociales: que facilitan los procesos didácticos, el trabajo con los jóvenes, la integración de las funciones formativas, la mentorización, el aprendizaje colaborativo y la trasferencia efectiva de conocimiento.

- Competencias de gestión: coordinación con agencias formativas colaboradoras y supervisión de prácticas.

Derivado de una investigación realizada (Álvarez, y otros, 2011) constataron que el profesorado percibe mayores carencias en la profesionalización docente, que en los aspectos disciplinares relacionados con su formación universitaria; sobre todo, con las competencias relacionadas con aspectos metodológicos centrado en el aprendizaje autónomo del alumnado más que la transmisión de los contenidos específicos de la ciencia. En este punto, de podría orientar el análisis también a lo planteado por la Comisión Europea (2005) citado en (Villardón, Concepción, Achurra, Iraurgi, y Aguilar, 2013) quien define otra competencia también necesaria, la 
competencia de aprendizaje, asumida como la habilidad de comenzar y continuar el aprendizaje individual, en cuanto a la regulación del tiempo y la información de forma efectiva, de manera autónoma o en grupo. Por otra parte, a decir de (Serrano y Pérez, 2018) también existen algunos aspectos del docente que se relaciona con lo social en los que resaltan las relaciones interpersonales y la cooperación con otras personas, dentro de los contextos educativos; así como las circunstancias socio-económicas que repercute directamente en sus estudiantes mediante su práctica docente y en su formación de la identidad.

Una de las estrategias metodológicas de interés actual por los docentes como respuesta a la evidente ineficiencia de la educación tradicional y de los sistemáticos cambios, surge el aprendizaje colaborativo que pretende la construcción social del conocimiento a favor de la transformación para satisfacer necesidades, proporcionar aprendizajes por competencias y desarrollar grupos de estudiantes, estudiante una manera formal e informal de organización de interese comunes de aprendizaje; a fin de propiciar creaciones propias con una condición activa, emprendedora y transformadora de su realidad (Barzola, Castillo, y Castillo, 2018). En correspondencia con lo antes dicho, este procedimiento puede constituir también una vía para el desarrollo de competencias docentes de la Educación Superior.

\section{Estrategia metodológica para el desarrollo de competencias docentes.}

Las competencias docentes constituyen la base fundamental para el correcto procedimiento en las aulas de cualquier nivel educativo, en tal sentido, el desarrollo de estrategias que propicien la calidad de la enseñanza-aprendizaje en el nivel de la Educación Superior siempre tributará al contexto social. No obstante, es importante que los procedimientos se encuentren dirigidos por estrategias concebidas con una base metodológica que ayuden tanto al docente inexperto como el experimentado. Por este motivo, es necesario asumir el significado de algunos términos, los cuales representan la base para el desarrollo de competencias docentes.

Según el Diccionario Pedagógico AMEI-WAECE citado en (Latorre y Seco, 2013).

Metodología es el conjunto de criterios y decisiones que organizan de forma global la acción didáctica en el aula, determinando el papel que juega el profesor, los estudiantes, la utilización de recursos y materiales educativos, las actividades que se realizan para aprender, la utilización del tiempo y especio, los agrupamientos de estudiantes, la 
secuenciación de los contenidos y los tipos y del espacio, los agrupamientos de estudiantes, las secuenciación de los contenidos y los tipos de actividades, etc. (p.16).

Por otro lado, estrategias es un término de procedencia militar, utilizado con el fin de proyectar, ordenar y dirigir sus operaciones para la esperada victoria mediante tácticas: pasos; por lo que constituye un procedimiento heurístico que propicia la resolución de problemas de aprendizaje de manera organizada y planificada (Latorre y Seco, 2013).

Existen varios métodos para apoyar el proceso de enseñanza-aprendizaje en cuanto a la investigación educativa de los docentes, entre ellas se encuentran las siguientes:

Cuadro 2. Métodos en la investigación educativa.

\begin{tabular}{|c|c|}
\hline MÉTODOS & DESCRIPCIÓN \\
\hline Clase magistral & $\begin{array}{l}\text { Comunica conocimientos y estimula procesos formativos de los } \\
\text { estudiantes, con habilidades desarrolladas en el área. Tiene un } \\
\text { orden didáctico, adaptación de los estudiantes, organización de } \\
\text { los contenidos y presentación de esquemas. }\end{array}$ \\
\hline $\begin{array}{c}\text { Trabajo personal o } \\
\text { aprendizaje autónomo }\end{array}$ & $\begin{array}{l}\text { Actividad individual de confrontación del estudiante con los } \\
\text { conocimientos, mediados por los constructos de asimilación y } \\
\text { adaptación de sus saberes con los nuevos conocimientos, } \\
\text { siempre y cuando esté presente la resolución de problemas } \\
\text { cognitivos para el aprendizaje significativo. }\end{array}$ \\
\hline $\begin{array}{c}\text { Aprendizaje colaborativo o } \\
\text { trabajo en equipo }\end{array}$ & $\begin{array}{l}\text { La actividad en un contexto social concreto, donde exista } \\
\text { interacción entre el estudiante con el docente o con sus } \\
\text { compañeros, mediante instrumentos o materiales de apoyo con } \\
\text { el fin de la asimilación adecuada de los contenidos. }\end{array}$ \\
\hline
\end{tabular}

Fuente: Elaboración propia a partir de Latorre y Seco (2013).

Existen otros enfoques relacionados con la metodología participativa según los referentes teóricos pedagógicos pueden encontrarse referenciada como "estrategia de enseñanza, estrategia de aprendizaje, estrategias o técnicas didácticas, estrategias docentes" (Farrach, 2016, 
p. 8). Lo que indica que pueden ser utilizados estos términos con diferentes significados y a su vez, diversas disquisiciones al respecto.

De cualquier forma, se podría pensar en una serie de estrategias metodológicas, para el desarrollo de competencias docentes mediante capacitaciones que aborden tanto dichos métodos como diferentes concepciones del aprendizaje basado en problemas, estudio de casos, clase magistral y seminario alemán; las cuales se presentan a continuación, para su correcta comprensión de la propuesta:

1. Aprendizaje basado en problemas: Aprendizaje por competencias de alto nivel de cognición para promover la "toma de decisiones, autonomía, habilidades de pensamiento crítico y resolución de problemas (...) interesantes, práctico y real resuelto en su día o todavía pendiente de resolver en un contexto real” (Sáez y Ruiz, 2012) p. 121.

2. Estudio de casos: Metodología apropiada para el diseño, implementación y evaluación, (...) aporta información válida tanto para el profesorado novel como para el experimentado" (López, Torrego, y Vallés, 2017).

3. Clase magistral: Estrategia apta para establecer técnicas basadas en aspectos lingüísticos, persuasivos, escenográficos y mediáticos, con el fin de transmitir información y lograr la retención de la misma en los estudiantes (Garcías, 2013).

4. Seminario alemán: Identifica los beneficios de la vida académica para trazarse metas sin aprovecharse de los demás tomando en consideración la estrategia de jerarquización, la escucha activa, trabajo en equipo y utilización de los recursos tecnológicos de manera adecuada, entre otros aspectos propios de la disciplina profesional (Pérez, 2010).

En este sentido, se proponen capacitaciones dirigidas a los docentes para desarrollar sus competencias en el marco de su práctica educativa; por ello a continuación se presentan estrategias metodológicas que pueden aplicar en su práctica docente, como forma de orientar la calidad de la educación superior:

Estrategia \# 1- Aprendizaje basado en problemas:

ISSN: 1390-9320, Edición Especial, diciembre 2019 
- Actividades del Docente: planteamiento de un problema asociado con la práctica laboral, según los perfiles laborales correspondientes, con el objetivo de establecer comparaciones entre de resoluciones entre los grupos presentes en el aula. De esta manera, un mismo problema puede solucionarse desde diferentes puntos de vista y se realizan asociaciones en cuanto a las alternativas de solución.

- Ejecución del Estudiante: explicación de los resultados del cumplimiento de la actividad, así como las dificultades presentadas durante el proceso para la búsqueda de alternativas de solución, con el fin de propiciar el debate significativo ante la toma de decisiones. De acuerdo con las premisas del respeto, enfoque crítico y profesional, se realizan apreciaciones sobre el contexto social y sus implicaciones económicas o medioambiental. Al final de esta etapa, se realiza la autoevaluación, coevaluación y evaluación de la ejecución para la adecuada retroalimentación del procedimiento.

\section{Estrategia \# 2- Estudio de casos:}

- Actividades del Docentes: utilización de ejemplos asociados con centros laborales cuyo desempeño vaya dirigido de acuerdo a los perfiles profesionales de los docentes, donde se hayan realizado procedimientos inadecuados desde diferentes puntos de vista (dependiendo de la ciencia) con el fin de establecer relaciones en comunes, sobre la comprensión de los conflictos que se presentan en el contexto laboral. Se orienta el trabajo individual y grupal sobre la búsqueda de referentes teóricos (fuentes de información) para su aplicación en la práctica con un enfoque interdisciplinario. En este sentido, es importante que se oriente correctamente las actividades con claridad y precisión, para su eficiente puesta en práctica por parte de los estudiantes.

- Ejecución del Estudiante: explicación de sus fortalezas y debilidades sobre la realización de la actividad, también abordarán la extrapolación de estas problemáticas a sus experiencias de vida propias o ajenas, con el fin de motivar a la reflexión sobre los valores éticos en la práctica laboral. 


\section{Estrategia \# 3- Clase magistral:}

- Actividades del Docentes: conferencista sobre una temática asociada con el área del conocimiento, con el fin de continuar profundizando en nuevas tendencias de investigación en relación con temas emergentes y autores reconocidos identificados en las fuentes de información de primera mano a nivel internacional. Estas acciones contribuyen a la autonomía del docente en la conformación de sus propias estrategias de proyección sobre el aprendizaje de la ciencia de interés, en cuanto a los procedimientos cognitivos y en función de ampliar sus experiencias como investigador.

- Ejecución del Estudiante: exposiciones de diferentes tipologías textuales orales formales (conferencias, disertación, ponencia en congreso) como forma de imitación del modelo del docente, para la producción del texto oral orientado a la aplicación del lenguaje científico y formal con base en la búsqueda de información válida y actualizada sobre temas de interés científico de manera que confirme la información presentada por el docente o contradiga los referentes teóricos con otros identificados por los estudiantes.

\section{Estrategia \# 4- Seminario alemán:}

- Actividades del Docentes: orientación de la redacción de informes de investigación, con el propósito de que los estudiantes gestionen su propio aprendizaje como científico en formación, para la aplicación de métodos de investigación en la búsqueda de soluciones válidas. En la aplicación de esta metodología, el docente debe ser consciente que los resultados de los estudiantes pueden no ser verdaderos y/u originales; ya que lo más importante es la práctica investigativa, que el alumno se sienta en un ambiente de confianza y seguridad en el desarrollo de su investigación, que le resulte interesante e importante para su futuro desempeño profesional.

- Ejecución del Estudiante: redacción de informes de investigación para la posterior puesta en común de las experiencias entre los integrantes de los grupos de trabajo, de esta manera, se propicia la retroalimentación y sistematización de los procedimientos investigativos; así como los obstáculos encontrados en el desarrollo de la actividad. La 
intención de esta estrategia es provocar discusiones saludables y críticas constructivas hacia la verdad de las soluciones, puesto que la respuesta a un problema de un grupo de estudiantes, puede ser motivo para que los integrantes de otros grupos mejoren las alternativas de solución y viceversa.

Estas cuatro propuestas antes mencionadas, son estrategias que pueden combinarse con otras en el desarrollo de la clase; ya que lo más importante es que el docente conozca de su existencia, y pueda implementarla en el salón en aras de contribuir a una práctica eficiente de su metodología. En las manos de los gestores de las capacitaciones dirigidas a los docentes se encuentra la responsabilidad de encontrar las estrategias apropiadas para orientar la aplicación de estas metodologías para una adecuada aplicación.

\section{CONCLUSIONES}

La revisión de los referentes teóricos en relación con la temática, representa un aspecto indispensable para el análisis del problema, en el conocimiento de la información pertinente para la selección de los procedimientos emergentes que propicien la creación de la propuesta como vía de solución.

Los docentes de la educación superior necesitan orientación durante el desarrollo de sus competencias docentes, por esta razón, es un compromiso de vital importancia contribuir a la búsqueda de estrategias que faciliten la alineación de actividades de capacitación ajustadas al contexto de desempeño de los académicos. Este interés parte de la necesidad de colaborar con el ejercicio pedagógico y contribuir a la motivación de los implicados en el proceso de la mejora de la calidad de este nivel educativo.

La utilización de la propuesta contribuye al análisis de los aspectos fundamentales del proceso pedagógico de los docentes universitarios, en relación con el desarrollo de sus competencias docentes, a fin de fortalecer la formación profesional y el futuro desempeño de los futuros profesionales de un país. 


\section{REFERENCIAS BIBLIOGRÁFICAS}

Álvarez, J., Chaparro, E. M., y Reyes, D. E. ( 2015). Estudio de la Satisfacción de los Estudiantes con los Servicios Educativos brindados por Instituciones de Educación Superior del Valle de Toluca. Revista Iberoamericana sobre, REICE. Vol. 13, Núm. 2, 5-26. Recuperado en: http://www.redalyc.org/articulo.oa?id=55138743001.

Álvarez, V., Soledad, Gil, J., Rodríguez, J., Clares, J., y Asensio, I. (2011). Necesidades de formación del profesorado universitario para la adaptación de su docencia al espacio europeo de educación superior (EEES). Revista Electrónica de Investigación y Evaluación Educativa. RELIEVE, Vol. 17, No. 1, art. 1., 1-22. Recuperado de: https://www.uv.es/RELIEVE/v17n1/RELIEVEv17n1_1eng.htm

Barzola, B., Castillo, M., y Castillo, C. (2018). Collaborative work as a methodological strategy in the learning of mathematics in the firt grade students of the National University Micaela Bastidas of Apurímac-Peru. 16th LACCEI International MultiConference for Engireering, Education, and Technology: "Innovation in Education and Inclusion. (DOI):http://dx.doi.org/10.18687/LACCEI2018.1.1.414, 1-10.

Enríquez, J. O. (2006). Educación superior: tendencias y desafíos. Educación médica, 9(1), 6-10. Recuperado de: http://scielo.isciii.es/pdf/edu/v9n1/colaboracion2.pdf

Farrach, G. A. (2016). Etrategias metodológicas para fometar la comprensión lectora. Revista Científica de FAREM-Estelí. Medio ambiente, tecnología y desarrollo humano. $N^{\circ} 20$ | Año 5, 5-19. DOI: https://doi.org/10.5377/farem.v0i20.3064

Fernandes, D., Sotolongo, M., y Martínez, C. C. (2016). La evaluación del Desempeño por Competencias: Percepciones de Docentes y Estudiantes en la Educación Superior. Formación universitaria., 15-24. Recuperado de: https://scielo.conicyt.cl/scielo.php?script=sci_abstract\&pid=S071850062016000500003\&l $\mathrm{ng}=\mathrm{es} \& \mathrm{nrm}=$ iso

Ferreyra, M. M., Avitabile, C., Botero, J., Hamovich, F., y Urzúa, S. (2017). Momento decisivo para la educación de América Latina y el Caribe. Washington DC: Resumen. Washington, DC: Banco Mundial. Licencia: Creative Commons. Recuperado de: https://openknowledge.worldbank.org/bitstream/handle/10986/26489/211014ovSP.pdf

García, J. M. (1999). Análisis multirrasgo-multimétodo en la validación de instrumentos para la evaluación d ela calidad docente en instituciones universitarias. Revista española de pedagogía., 417-444. Recuperado de: https://revistadepedagogia.org/wpcontent/uploads/2007/06/1Analisis_multirrasgomiltimet odo_en_la_validacon_instrumentos.pdf

Garcías, D. (2013). El Think Different de las clases magistrales. Estudio sobre el mensaje periordístico, 19, 773-782. 
González, J., y Santamaría, R. (2013). Calidad y acreditación en la educación superior: integración e internacionalización de América Latina y el Caribe. Educación Vol. $X X I I, N^{\circ} 43,132-147$ Recuperado de revistas.pucp.edu.pe/index.php/educacion/article/view/7500/7739.

Guzmán, J. C. (2011). La calidad de la enseñanza en educación superior ¿Qué es una buena enseñanza en este nivel educativo? Perfile educativos, vol.33.

Jiménez, A., Palmero, C., y Jiménez, A. (2012). El impacto de la educación secundaria y superior en la creación de empress en la Unión Europea. Revista española de pedagogía., 201-219.

Lago, D., Gamoba, A. A., y Montes, A. J. (2014). Calidad de la educación superior: un análisis de sus principales determinantes. Saber, ciencia y libertad, Vol. 8, No. 2 , 157-169.

Latorre, M., \& Seco, C. J. (2013). Metodología. Estrategia y técnicas metodológicas. Lima, Perú: Universidad Marcelo Champagnat ISBN No: 978-9972-9739-6-3.

López, M., Torrego, L., y Vallés, C. (2017). Una Investigación Mediante Estudio de Casos en la Formación Científica de docentes de educación Infantil. Investigación cualitativa en Investigación, 1, 580-589.

Martelo, R., Rodríguez, J., y Villabona, N. (2018). Guía metodológica para determinar prácticas pedagógicas de docentes en instituciones de educación superior. Revista Espacios, Vol. 39, No. 5 ISSN 07981015, 1-13.

Martin, J. F. (2018). Calidad educativa en la educación superior colombiana: una aproximación teórica. Sophia, 14 (2), 4-14.

Martínez, J. E., Tobón, S., y Romero, A. (2017). Problemáticas relacionadas con la acreditación de la calidad de la educación superior en América Latina. Innovación educativa (México, DF) Vol.17, No.73. Recuperado de:

http://www.scielo.org.mx/scielo.php?pid=S1665-

26732017000100079\&script=sci_arttext\&tlng=pt.

Murillo, F. J. (2011). Mejora de la eficiencia escolar en Iberoamérica. Revista IBEROAMERICANA DE EDUCAÇÃO. N. ${ }^{\circ}$ 55., 49-83.

Nemiña, R. E., García, H. M., y Montero, L. (2009). Desarrollo profesional y profesionalización docente. Perspectivas y problemas. Profesorado. Revista de Currículum y Formación de Profesorado, Vol. 13, Núm. 2, 1-13. Recuperado de: http://www.redalyc.org/articulo.oa?id=56711798016.

Orozco, L. E. (2010). Calidad académica y relevancia social de la educación superior en América Latina. Revista Iberoamericana de educación superior. Vol. 1, No. 1, 24-36 Recuperado de: http://www.scielo.org.mx/pdf/ries/v1n1/v1n1a3.pdf. 
Ortega, R., y González, K. (2017). Calidad en la enseñanza en educación superior del Centro Universitario del Norte, Universidad de Guadalajara, México. Revista Iberoamericana de Educação, Vol. 74, Núm. 1, 9-22.Recuperado de: https://rieoei.org > historico > documentos

Palominos, F. E., Mendez, M. A., y Barrera, R. A. (2014). Sistema de Perfeccionamiento Orientado a Competencias para Docentes de la Educación Superior. Formación universitaria., 11-22. Recuperado de: https://scielo.conicyt.cl > scielo > pid=S071850062014000300003

Pérez, J. (2010). El seminario alemán una estrategia pedagógica para el estudiante. Cultura, educación y sociedad, 1(1), 107-112. Recuperado de: https://revistascientificas.cuc.edu.co > article > download > pdf_138

Pozo, C., Bretones, B., Martos, M. J., y Alonso, E. (2011). Evaluación de la actividad docente en el Espacio Europeo de Educación Superior: un estudio comparativo de indicadores de calidad en universidades europeas. Revista española de pedagogía , 145-163. Recuperado de: https://revistas.ucm.es > index.php > RCED > article > view

Quiroz, E. ( 2007). Competencias profesionales y calidad en la educación superior. Núm. 50, 93-99. Recuperado de: http://www.redalyc.org/articulo.oa?id=34005012.

Sáez, J. M., y Ruiz, J. M. (2012). Estrategias metodológicas, aprendizaje colaborativo y TIC: un caso en la Escuela Computense Latinoamericana. Revista Computence de Educación. Vol. 23, Núm. 1, 115-134. Recuperado de : http://dx.doi.org/10.5209/rev_RCED.2012.v23.n1.39105

Sánchez, J., Chávez, J., y Mendoza, J. (2018). La calidad en la educación superior: una mirada al proceso de evaluación y acreditación de universidades del Ecuador. Revista Caribeña de Ciencias Sociales, [En Línea] Recuerado de: http://www.eumed.net/rev/caribe/2018/01/calidad-educacion-superior.html.

Sandoval, F. (29 de mayo de 2017). El acceso a la educación superior aumentó en toda América Latina. Diario El Telégrafo. Recuperado de: https://www.eltelegrafo.com.ec/noticias/sociedad/6/el-acceso-a-la-educacion-superioraumento-en-toda-america-latina.

Serrano, R., y Pérez, E. (2018). La motivación, la formación psicopedagógica y el practicum facilitadores del desarrollo de la identidad profesional docente. Revista científica ECOCIENCIA, Vol. 5, No. 6. Consultado 7 de junio de 2019. Recuperado de: http://ecociencia.ecotec.edu.ec/articulo?ida=147, 1-21.

Silander, C., y Stigmar, M. (2018). Individual growth or institutional development? High Educ, https://doi.org/10.1007/s10734-018-0272-z.

Tejada, J. (11 de abril de 2013). Profesionalización docente en la universidad: implicaciones desde la formación. Recuperado de La informalización de la educación: <http://rusc.uoc.edu/ojs/index.php/rusc/article/view/v10n1-tejada/v10n1-tejada-es> 
Tünnermann, C. (2010). Las conferencias regionales y mundiales sobre educación de Educación Superior de la Unesco y su impacto en la Educación Superior de América Latina. Unión de Universidades de América Latina y el Caribe, núm. 47, pp. 31-46 Recuperado de https://www.redalyc.org/pdf/373/37318570005.pdf.

UNESCO. (1998). La educación superior en el siglo XXI: Visión y acción. Recuperado de http://www.unesco.org/education/educprog/wche/declaration_spa.htm.

Villardón, L., Concepción, Y., Achurra, C., Iraurgi, O., y Aguilar, M. C. ( 2013). Learning Competence in University: Development and Structural Validation. Revista de Psicodidáctica, 18(2)., 357-374 DOI: 10.1387/RevPsicodidact.6470. 